\section{Malnutrition risk prevalence and clinical outcomes among acute hospital inpatients in Hong Kong}

Terry HY Ting ${ }^{1}$, MSc, CY Wong ${ }^{1}$, MSc, Sherry YK Ng${ }^{2}$, B (Health Science [Nursing]), M (Applied Management [Nursing]), Sammei OF Tam ${ }^{3}$, MSc (Nursing), MSc (Health and Hospital Management)

\section{ORIGINAL ARTICLE}

\footnotetext{
Dietetics Department, North District Hospital, New Territories, Hong Kong

Department of Surgery, North District Hospital, New Territories, Hong Kong

3 Central Nursing Department, North District Hospital, New Territories, Hong Kong
}

Methods. Records of 40,105 adult patients admitted to our hospital between January and December 2016 were retrieved. We recorded patient Malnutrition Screening Tool (MST) scores (range, 0-5) and outcomes after discharge from hospital such as length of hospital stay (LOS), 28-day emergency readmission rate, and death. The malnutrition risk was categorised into three levels according to MST score: low risk (0-1), medium risk (2), and high risk ( $\geq 3$ ). We made comparisons among the three groups, and between the low-risk group and the mediumand high-risk (at-risk) group.

Results. The prevalence of malnutrition risk was $9.2 \%$. Malnutrition risk was $12.0 \%$ among those aged $\geq 65$ years and $6.1 \%$ among those aged 18 to 65 years. The low-, medium- and high-risk groups differed significantly in terms of the mean patient age (64.2 vs. 70.8 vs. 73.7 years, $\mathrm{p}<0.01)$ and the mean LOS (4.2 vs. 6.4 vs. 7.4 days, $\mathrm{p}<0.001$ ). The emergency readmission rate within 28 days was higher in at-risk patients than in low-risk patients $(25.1 \%$ vs. $14.6 \%$, odds ratio $=2.0$, $\mathrm{p}<0.001)$, as was the mortality rate $(8.5 \%$ vs. $2.3 \%$, odds ratio $=3.9$, $\mathrm{p}<0.001)$.

Conclusion. Malnutrition is a common problem among hospitalised patients. The use of a validated malnutrition screening tool to lower the malnutrition risk is necessary. A multicentre cross-sectional study on the effect of malnutrition on clinical outcomes is needed for developing service-quality enhancement measures.

Correspondence to: Dr Terry HY Ting, 1/F, Dietetics Department, North District Hospital, New Territories, Hong Kong. Email: tinghoyan@gmail.com

\section{INTRODUCTION}

Malnutrition is defined as a state of nutrition in which an excess or deficiency of energy, macro- and micro-nutrients causes adverse effects on clinical, functional, and economic outcomes. ${ }^{1}$ Malnutrition is common in health care settings, particularly in an acute care hospitals, when patients reduce their food intake owing to neurological disorders (including dysphagia) that decrease the ability to self-feed, sideeffects of drugs or treatments, or deterioration in taste and smell leading to a poor appetite. ${ }^{2}$ Malnutrition is more likely to develop in older adults with comorbid chronic conditions, impaired adaptation to inflammatory-catabolic states, or a heightened risk of acute diseases. ${ }^{3}$ By 2050, the number of older 
adults worldwide is set to triple from 605 million to 2 billion; a comprehensive system is needed to prevent malnutrition and associated adverse consequences. ${ }^{4}$

The prevalence of malnutrition has been reported to be $20 \%$ to $50 \%$ among hospitalised patients, ${ }^{5-7}$ but the sample size in most studies was small. In Hong Kong, two studies more than 10 years ago reported the prevalence of malnutrition among geriatric and stroke patients., ${ }^{2,8}$ The prevalence was likely underreported because of failure to recognise malnutrition in inpatients $(60 \%$ to $85 \%)$ and outpatients $(64 \%$ to $73 \%) .{ }^{9}$ Malnutrition is associated with adverse clinical and functional outcomes. Length of hospital stay (LOS) is 1.5- to 2.0-times longer in malnourished patients than in well-nourished patients, after controlling for confounding factors. ${ }^{10-12}$ The mortality rate is higher in undernourished inpatients than in those who were not undernourished $(66.7 \%$ vs. $33.3 \%) .{ }^{11}$ The mortality rate of post-discharge malnourished patients is 3-times higher than that of wellnourished patients. ${ }^{10}$ Malnutrition is positively associated with readmission rates. The risk of readmission within 15 days of discharge is $60 \%$ higher for malnourished patients compared with well-nourished patients. ${ }^{10}$ Malnourished patients are 1.5-times more likely to be readmitted within 6 months. ${ }^{13}$ Malnutrition is also associated with complications such as wound dehiscence and infections that lead to increased hospital readmission and LOS. ${ }^{14}$

The cost of hospitalisation for malnourished patients increases by $24 \%$ to $61 \% .^{10,15}$ The extra cost is mostly attributed to a longer LOS owing to increased infection rates and impaired wound healing and thus increased readmissions. ${ }^{10}$ Many international organisations, agencies, and governments recommend routine screening of malnutrition in hospital patients. ${ }^{9}$ Malnutrition can be readily treated at a relatively low cost, and nutritional screening can potentially reduce inappropriate referrals. It is suggested that the reductions in malnutritioninduced costs may far outweigh the cost of nutrition screening. ${ }^{9}$ Indeed, nutrition screening has long been the standard of care for patients admitted to hospitals in most countries.

A validated malnutrition screening tool can help identify high-risk patients for early intervention. ${ }^{16}$
The effectiveness of screening tools is maximised if used appropriately, based on the settings, target population, and local resources. For example, the Malnutrition Universal Screening Too ${ }^{17}$ is suitable for adults in a community setting, whereas the Simplified Nutritional Appetite Questionnaire ${ }^{18}$ is useful in predicting weight loss in institutionalised older adults. The Nutrition Risk Screening and the Mini Nutritional Assessment consider body mass index. ${ }^{19,20}$ The Malnutrition Screening Tool (MST) is widely used in hospital settings because it has been validated among adult inpatients and considers current and past weight status of patients, linking to their appetite..$^{21}$ The MST is a 2-question screening tool with scores ranging from 0 to 5. Questions are about weight loss history and recent oral intake amount.

Although well reported internationally, local research on how hospital malnutrition affects the clinical outcomes is limited. ${ }^{1-4}$ Therefore, this crosssectional study aimed to determine the malnutrition prevalence and clinical outcomes of patients at an acute hospital in Hong Kong.

\section{METHODS}

This study was approved by the Joint Chinese University Hong Kong-New Territories East Cluster Clinical Research Ethics Committee. The North District Hospital is a 600-bed acute district hospital serving approximately 315,270 people. The hospital has used an electronic patient assessment form since 2016. Nursing staff are required to use the MST to screen all adults admitted to the hospital within 48 hours. The MST was introduced to our hospital in 2011. Training and refreshment briefings on how to use the MST are provided to frontline nurses on a regular basis. The Clinical Management System is used to record patient data, including MST scores and outcomes after discharge such as the LOS, 28day emergency readmission rate, and death.

Adult patients were categorised into three groups according to malnutrition risk levels (MST score): low risk (0-1), medium risk (2), and high risk ( $\geq 3)$ [TABLE 1]. We made comparisons among the three groups, and between the low-risk group and the medium- and high-risk (at-risk) group. For patients with cognitive impairment or dementia, their caregivers, relatives, or records from their care home 
were queried, according to the guideline Nutrition Care for Patient with Malnutrition Risk.

Records of 40,105 adult patients admitted between January and December 2016 were retrieved from the Hospital Authority Clinical Data Analysis and Reporting System. The low-risk group and the at-risk group were compared using the independent t-test and one-way analysis of variance for the LOS, and using the Pearson Chi-squared test for the readmission rate and mortality rate. A $p$ value of $<0.05$ was considered statistically significant. Statistical analyses were performed using SPSS (Windows version 21; IBM Corp, Armonk [NY], US).

\section{RESULTS}

Patients were admitted to the medical (42\%), surgical (37\%), accident and emergency (11\%), or orthopaedic (10\%) departments. $54 \%$ of patients were men. The mean patient age was 64.9 (standard deviation, 18.5; range, $18-109$ ) years; $47.6 \%$ of patients were aged 18 to 65 years.

TABLE 1

Malnutrition Screening Tool ${ }^{20}$

\begin{tabular}{lc}
\hline Malnutrition Screening Tool & Total score \\
\hline $\begin{array}{l}\text { How much weight }(\mathrm{kg}) \text { have you lost } \\
\text { recently without trying? }\end{array}$ & Weight loss score \\
No & 1 \\
$1-5$ & 2 \\
$6-10$ & 3 \\
$11-15$ & 4 \\
$>15$ & 2 \\
Unsure & Appetite score \\
Have you been eating poorly because of a \\
decreased appetite?
\end{tabular}

Among patients in the medical department, $14.1 \%$ were at risk of malnutrition $(6.1 \%$ at medium risk and $8.0 \%$ at high risk). Among patients in the surgical department, $7.3 \%$ were at risk of malnutrition ( $4.2 \%$ at medium risk and $3.1 \%$ at high risk). Among patients in the accident and emergency department, $3.6 \%$ were at risk of malnutrition $(2.1 \%$ at medium risk and $1.5 \%$ at high risk). Among patients in the orthopaedic department, $2.4 \%$ were at risk of malnutrition (1.4\% at medium risk and $0.9 \%$ at high risk) [TABLE 2].

The prevalence of malnutrition risk was 9.2\% ( $4.5 \%$ at medium risk and 4.7 at high risk); it was $12.0 \%$ among those aged $\geq 65$ years and $6.1 \%$ among those aged 18 to 65 years. Older patients were at higher risk of malnutrition; the mean patient age for the low-, medium- and high-risk groups differed significantly (64.2 vs. 70.8 vs. 73.7 years, $p<0.01$, Table 3). Among women, $89.9 \%$ were at low risk, $4.4 \%$ at medium risk, and $5.7 \%$ at high risk. Among men, $91.6 \%$ were at low risk, $4.5 \%$ at medium risk, and $3.9 \%$ at high risk.

The LOS was longer in patients with higher risk of malnutrition; the mean LOS differed significantly among the low-, medium-, and high-risk groups (4.2 vs. 6.4 vs. 7.4 days, $\mathrm{p}<0.001$, TABle 3). Compared with low-risk patients, the LOS was $76 \%$ longer in high-risk patients, $52 \%$ longer in medium-risk patients, and $64.2 \%$ longer in at-risk (medium and high risk) patients.

The emergency readmission rate within 28 days was higher in at-risk patients than low-risk patients $\left(25.1 \%\right.$ vs. $14.6 \%$, odds ratio $=2.0, \chi^{2}=280.43, p<0.001$, TABLE 3).

The mortality rate was higher in at-risk patients than low-risk patients ( $8.5 \%$ vs. $2.3 \%$, odds ratio $=3.9$, $\chi^{2}=445.90, \mathrm{p}<0.001$, TABLE 3).

TABLE 2

Prevalence of malnutrition risk in different specialty departments

\begin{tabular}{lccccc}
\hline Malnutrition risk & \multicolumn{5}{c}{ Prevalence (\%) } \\
\cline { 2 - 6 } & $\begin{array}{c}\text { Medical } \\
(\mathrm{n}=16,559)\end{array}$ & $\begin{array}{c}\text { Surgical } \\
(\mathrm{n}=15,225)\end{array}$ & $\begin{array}{c}\text { Orthopaedic } \\
(\mathrm{n}=4023)\end{array}$ & $\begin{array}{c}\text { Accident \& } \\
\text { emergency } \\
(\mathrm{n}=4298)\end{array}$ & $\begin{array}{c}\text { Overall } \\
(\mathrm{n}=40,105)\end{array}$ \\
\hline High & 8.0 & 3.1 & 0.9 & 1.5 & 4.7 \\
Medium & 6.1 & 4.2 & 1.4 & 2.1 & 4.5 \\
Low & 85.9 & 92.7 & 97.6 & 96.4 & 90.8 \\
\hline
\end{tabular}


TABLE 3

Comparison of patients at low, medium, and high risk of malnutrition*

\begin{tabular}{|c|c|c|c|c|c|c|}
\hline & \multirow[t]{3}{*}{ All $(n=40,105)$} & \multicolumn{3}{|c|}{ Malnutrition risk } & \multirow[t]{3}{*}{ Odds ratio } & \multirow[t]{3}{*}{$p$ Value } \\
\hline & & \multirow[t]{2}{*}{ Low risk $(n=36,417)$} & \multicolumn{2}{|c|}{ At risk } & & \\
\hline & & & $\begin{array}{l}\text { Medium risk } \\
(n=1793)\end{array}$ & High risk $(n=1895)$ & & \\
\hline Age, y & $64.9 \pm 18.5(18-109)$ & $64.2 \pm 18.5(18-109)$ & $70.8 \pm 16.8(18-109)$ & $73.7 \pm 15.6(19-103)$ & - & $<0.01$ \\
\hline Women & $18,449(46.0)$ & 16,588 (89.9) & $817(4.4)$ & $1044(5.7)$ & - & - \\
\hline Men & $21,656(54.0)$ & $19,829(91.6)$ & $976(4.5)$ & $851(3.9)$ & - & - \\
\hline Length of hospital stay, $d$ & $4.4 \pm 6.9$ & $4.2 \pm 6.5$ & $6.4 \pm 9.0$ & $7.4 \pm 10.8$ & - & $<0.001$ \\
\hline Readmission within $28 \mathrm{~d}$ & $6218(15.4)$ & $5294(14.6)$ & \multicolumn{2}{|c|}{$924(25.0)$} & 2.0 & $<0.001$ \\
\hline Mortality & $1163(2.9)$ & 851 (2.3) & \multicolumn{2}{|c|}{312 (8.5) } & 3.9 & $<0.001$ \\
\hline
\end{tabular}

* Data are presented as mean \pm standard deviation (range), mean \pm standard deviation, or no. (\%) of patients

\section{DISCUSSION}

Among inpatients in the present study, the prevalence of malnutrition risk was $9.2 \%(4.5 \%$ at medium risk and $4.7 \%$ at high risk), which is lower than the $20 \%$ to $85 \%$ reported in other studies. ${ }^{5-8}$ Among patients in the medical department, 14\% were at risk of malnutrition, which is lower than the $24 \%$ to $69 \%$ reported in other studies. ${ }^{22-25}$ In a study of 850 accident and emergency patients, the prevalence of malnutrition was $20 \%,{ }^{26}$ which is higher than the $3.6 \%$ reported in our accident and emergency patients. Among patients in the surgical and orthopaedic departments, the prevalence of malnutrition risk was $7.3 \%$ and $2.5 \%$, respectively, which is generally lower than the $5.5 \%$ to $57 \%$ reported in other studies. ${ }^{24,25,27}$

One possible reason for the differences in prevalence among studies is the use of different screening tools. The MST was used in the present study, whereas the Mini Nutritional Assessment, Malnutrition Universal Screening Tool, Nutrition Risk Screening, and Simplified Nutritional Appetite Questionnaire were used other studies. There are significant discrepancies in the malnutrition prevalence estimated by different screening tools. ${ }^{27-29} \mathrm{~A}$ large age range can be another reason for the low prevalence of malnutrition risk in our patients. Elderly patients are more likely to have malnutrition. ${ }^{23,30,31}$ In the present study, patients aged 18 to 65 years accounted for $47.6 \%$ of all patients, and their prevalence of malnutrition risk was $6.1 \%$, which was half that of the elderly patients (12.0\%).
In addition, the weight loss aspect of the MST score may underestimate the prevalence of malnutrition, because it was developed for Western populations and may not be accurate for Chinese populations owing to smaller body frames. For example, a 40$\mathrm{kg}$ woman losing $4 \mathrm{~kg}(10 \%)$ would receive an MST score of 0 , but this is substantial weight change for a woman with small body frame, and unintentional loss of $>10 \%$ of weight in 6 months is one of the diagnostic criteria for undernutrition. ${ }^{32}$

Elderly patients are at higher risk of malnutrition. ${ }^{23,30,31}$ Patients with higher malnutrition risk have 1.5- to 2-times longer LOS than wellnourished patients. ${ }^{10,11}$ Patients with malnutrition have longer LOS because of their medical or surgical problems. ${ }^{33,34}$ Among at-risk patients in the present study, the readmission rate and mortality rate were 2.0- and 3.9-times higher, respectively, compared with low-risk patients. This finding is similar to that reported in other studies. . $^{1011,13,14}$

One limitation of this study is the narrow patient population that included only four specialty departments. Findings of this study may not be generalised to other specialty patients in acute and sub-acute hospitals. In addition, the MST score has not yet been validated in Asian or Chinese populations. Reasons for losing weight were not recorded for further analysis. Dietetic assessment was not performed to confirm the malnutrition. Confounding factors (disease severity and type) that may affect the LOS, readmission, and mortality were not investigated. 


\section{CONCLUSION}

Malnutrition is a common risk problem among hospitalised patients. The use of a validated malnutrition screening tool to lower the malnutrition risk is necessary. A multicentre cross-sectional study on the effect of malnutrition on clinical outcomes is needed for developing service-quality enhancement measures.

\section{DECLARATION}

The authors have no conflict of interest to disclose.

\section{REFERENCES}

1. O'Shea E, Trawley S, Manning E, Barrett A, Browne V, Timmons S. Malnutrition in hospitalised older adults: a multicentre observational study of prevalence, associations and outcomes. J Nutr Health Aging 2017;21:830-6. Crossref

2. Shum NC, Hui WW, Chu FC, Chai J, Chow TW. Prevalence of malnutrition and risk factors in geriatric patients of a convalescent and rehabilitation hospital. Hong Kong Med J 2005;11:234-42.

3. Cereda E, Pedrolli C, Klersy C, et al. Nutritional status in older persons according to healthcare setting: a systematic review and meta-analysis of prevalence data using MNA®. Clin Nutr 2016;35:1282-90. Crossref

4. World Health Organization. Interesting facts about ageing. 2012. Available from: www.who.intageingabout facts en index.html. pdf. Accessed 1 June 2018.

5. Garcia RS, Tavares LR, Pastore CA. Nutritional screening in surgical patients of a teaching hospital from Southern Brazil: the impact of nutritional risk in clinical outcomes. Einstein (Sao Paulo) 2013;11:147-52. Crossref

6. Vedantam A, Subramanian V, Rao NV, John KR. Malnutrition in free-living elderly in rural south India: prevalence and risk factors. Public Health Nutr 2010;13:1328-32. Crossref

7. Tsai AC, Ho CS, Chang MC. Assessing the prevalence of malnutrition with the Mini Nutritional Assessment (MNA) in a nationally representative sample of elderly Taiwanese. J Nutr Health Aging 2008;12:239-43. Crossref

8. Chai J, Chu FC, Chow TW, Shum NC. Prevalence of malnutrition and its risk factors in stroke patients residing in an infirmary. Singapore Med J 2008;49:290-6.

9. Elia M, Zellipour L, Stratton RJ. To screen or not to screen for adult malnutrition? Clin Nutr 2005;24:867-84. Crossref

10. Lim SL, Ong KC, Chan YH, Loke WC, Ferguson M, Daniels L. Malnutrition and its impact on cost of hospitalization, length of stay, readmission and 3-year mortality. Clin Nutr 2012;31:34550. Crossref

11. Leiva Badosa E, Badia Tahull M, Virgili Casas N, et al. Hospital malnutrition screening at admission: malnutrition increases mortality and length of stay. Nutr Hosp 2017;34:907-13. Crossref

12. Middleton MH, Nazarenko G, Nivison-Smith I, Smerdely P. Prevalence of malnutrition and 12-month incidence of mortality in two Sydney teaching hospitals. Intern Med J 2001;31:45561. Crossref

13. Planas M, Audivert S, Pérez-Portabella C, et al. Nutritional status among adult patients admitted to an university-affiliated hospital in Spain at the time of genoma. Clin Nutr 2004;23:101624. Crossref

14. Bohl DD, Shen MR, Mayo BC, et al. Malnutrition predicts infectious and wound complications following posterior lumbar spinal fusion. Spine (Phila Pa 1976) 2016;41:1693-9. Crossref

15. Correia MI, Waitzberg DL. The impact of malnutrition on morbidity, mortality, length of hospital stay and costs evaluated through a multivariate model analysis. Clin Nutr 2003;22:2359. Crossref

16. Tappenden KA, Quatrara B, Parkhurst ML, Malone AM, Fanjiang G, Ziegler TR. Critical role of nutrition in improving quality of care: an interdisciplinary call to action to address adult hospital malnutrition. J Acad Nutr Diet 2013;113:1219-37. Crossref

17. Malnutrition Advisory Group. Guidelines for detection and management of malnutrition. Redditch, UK: British Association for Parenteral and Enteral Nutrition; 2000.

18. Wilson MM, Thomas DR, Rubenstein LZ, et al. Appetite assessment: simple appetite questionnaire predicts weight loss in community-dwelling adults and nursing home residents. Am J Clin Nutr 2005;82:1074-81. Crossref

19. GuigozY,Vellas B, Garry PJ. Assessing the nutritional status of the elderly: The Mini Nutritional Assessment as part of the geriatric evaluation. Nutr Rev 1996;54:S59-65. Crossref

20. Gheorge L, Iacob S. ESPEN guidelines for evaluating nutritional status 2002 [in Romanian]. Rom J Gastroenterol 2004;13:66-73.

21. Ferguson M, Capra S, Bauer J, Banks M. Development of a valid and reliable malnutrition screening tool for adult acute hospital patients. Nutrition 1999;15:458-64. Crossref

22. Singh H, Watt K, Veitch R, Cantor M, Duerksen DR. Malnutrition is prevalent in hospitalized medical patients: are housestaff identifying the malnourished patient? Nutrition 2006;22:3504. Crossref

23. Pirlich M, Schütz T, Kemps M, et al. Prevalence of malnutrition in hospitalized medical patients: impact of underlying disease. Dig Dis 2003;21:245-51. Crossref

24. Barker LA, Gout BS, CroweTC. Hospital malnutrition: prevalence, identification and impact on patients and the healthcare system. Int J Environ Res Public Health 2011;8:514-27. Crossref

25. Pirlich M, Schütz T, Norman K, et al. The German hospital malnutrition study. Clin Nutr 2006;25:563-72. Crossref

26. Edington J, Boorman J, Durrant ER, et al. Prevalence of malnutrition on admission to four hospitals in England. The Malnutrition Prevalence Group. Clin Nutr 2000;19:191-5. Crossref

27. Rasmussen HH, Kondrup J, Staun M, Ladefoged K, Kristensen $\mathrm{H}$, Wengler A. Prevalence of patients at nutritional risk in Danish hospitals. Clin Nutr 2004;23:1009-15. Crossref

28. Velasco C, García E, Rodríguez V, et al. Comparison of four nutritional screening tools to detect nutritional risk in hospitalized patients: a multicentre study. Eur J Clin Nutr 2011;65:26974. Crossref

29. Neelemaat F, Meijers J, Kruizenga $H$, van Ballegooijen $H$, van Bokhorst-de van der Schueren M. Comparison of five malnutrition screening tools in one hospital inpatient sample. J Clin Nurs 2011;20:2144-52. Crossref

30. Burns JT, Jensen GL. Malnutrition among geriatric patients admitted to medical and surgical services in a tertiary care hospital: frequency, recognition, and associated disposition and reimbursement outcomes. Nutrition 1995;11(2 Suppl):245-9.

31. Compan B, di Castri A, Plaze JM, Arnaud-Battandier F. Epidemiological study of malnutrition in elderly patients in acute, sub-acute and long-term care using the MNA. J Nutr Health Aging 1999;3:146-51.

32. Hoffbrand BI. Book reviews: Treat Obesity Seriously. A Clinical Manual. Postgrad Med J 1982;58:671. Crossref

33. Gallagher-Allred CR, Voss AC, Finn SC, McCamish MA. Malnutrition and clinical outcomes: the case for medical nutrition therapy. J Am Diet Assoc 1996;96:361-9. Crossref

34. Chima CS, Barco K, Dewitt ML, Maeda M, Teran JC, Mullen KD. Relationship of nutritional status to length of stay, hospital costs, and discharge status of patients hospitalized in the medicine service. J Am Diet Assoc 1997;97:975-80. Crossref 